\title{
BROWNIAN MOTION ON A SMASH LINE
}

\author{
Demosthenes Ellinas 周 and Ioannis Tsohantjis 9 \\ Department of Sciences \\ Technical University of Crete \\ GR-73 100 Chania Crete Greece
}

\begin{abstract}
Brownian motion on a smash line algebra (a smash or braided version of the algebra resulting by tensoring the real line and the generalized paragrassmann line algebras), is constructed by means of its Hopf algebraic structure. Further, statistical moments, non stationary generalizations and its diffusion limit are also studied. The ensuing diffusion equation posseses triangular matrix realizations.
\end{abstract}

\footnotetext{
†Submitted to Journal of Nonlinear Mathematical Physics. Special Issue of Proccedings of NEEDS'99.

* Email: ellinas@science.tuc.gr

घ Email: ioannis2@otenet.gr
} 
Introduction. The study of random walks and diffusions on generalized spaces is an active field of research. The formulation of the related problems is carried out mostly in a algebraic manner that employs commuting and/or non-commuting algebras with rich structures such as bialgebras and Hopf algebras with possible braiding or smashing defined among them cf. [1, 2, 3]. Here we construct and study further such a Brownian motion on a smash line algebra. Our algebraic model for the braided/smash line is the merging of the $*$-Hopf algebra of formal power series $\mathcal{A}=\mathbf{C}[[x]]$ on $\mathbf{R}$, with the braided $*$-Hopf algebra $\mathcal{B}=\mathbf{C}[\xi] / \xi^{N}$, of $(N-1)$-degree polynomials generated by the $N$-potent variable $\xi(N=2$ gives us the standard supespace). Technically this merging is the cross-product (also known as smash-product) of $\mathcal{A}, \mathcal{B}$ algebras. It imposes a definite braiding rule between $\mathcal{A}, \mathcal{B}$ that is naturally interpreted as non commutativity among the increments (steps) of the underlying random walk. Once a natural positive definite functional has been chosen on the smash line we compute the values of the statistical moments of any order for our $(x, \xi)$ random variables. Introducing an appropriate limiting procedure we construct the (pseudo)differential equation of the diffusion on the smash line. This is further extended to non stationary random walks that incorporate Hamiltonian dynamics into the diffusions processes. Additionally a matrix realizations of the $\xi$-generators of the $\mathcal{B}$ algebra and of their differential are employed to cast the resulting diffusion equation into the form of a matrix-valued (ordinary)differential equation that gives rise to a system of coupled diffusion equations.

Smash line algebra. Let us consider two complex associative algebras: $\mathcal{A}=\mathbf{R}[[x]]$, the algebra of real formal power series in one variable generated by the element $x$ with $\left\{1, x, x^{2}, \ldots\right\}$ as a linear basis, and $\mathcal{B}=\mathbf{R}[\xi] / \xi^{N}$ the algebra of polynomials in one variable of degree $N-1$, generated by the element $\xi$ with a basis $\left\{1, \xi, \xi^{2}, \ldots \xi^{N-1}\right\}$ and $\xi^{N}=0$. Both these algebras are equipped with a Hopf algebra structure. Algebras $\mathcal{A}=\mathcal{A}\left(\mu_{\mathcal{A}}, \Delta_{\mathcal{A}}, u_{\mathcal{A}}, \varepsilon_{\mathcal{A}}\right), \mathcal{B}=\mathcal{B}\left(\mu_{\mathcal{B}}, \Delta_{\mathcal{B}}, u_{\mathcal{B}}, \varepsilon_{\mathcal{B}}\right)$ are commuting, cocommuting coassociative algebras with products $\mu_{\mathcal{A}}, \mu_{\mathcal{B}}$, coproducts, $\Delta_{\mathcal{A}}, \Delta_{\mathcal{B}}$, units $u_{\mathcal{A}}, u_{\mathcal{B}}$, and counits $\varepsilon_{\mathcal{A}}, \varepsilon_{\mathcal{B}}$ respectively defined as $\mu_{\mathcal{A}}(x \otimes y)=x y, \mu_{\mathcal{B}}(\xi \otimes \eta)=\xi \eta, u_{\mathcal{A}}(c)=c \mathbf{1}_{\mathcal{A}}, u_{\mathcal{B}}(c)=c \mathbf{1}_{\mathcal{B}}, c \in \mathbf{C}$, $\Delta_{\mathcal{A}}(x)=x \otimes \mathbf{1}_{\mathcal{A}}+\mathbf{1}_{\mathcal{A}} \otimes x, \Delta_{\mathcal{B}}(\xi)=\xi \otimes \mathbf{1}_{\mathcal{B}}+\mathbf{1}_{\mathcal{B}} \otimes \xi, \epsilon_{\mathcal{A}}(x)=0, \epsilon_{\mathcal{A}}\left(\mathbf{1}_{\mathcal{A}}\right)=1, \varepsilon_{\mathcal{B}}(\xi)=0$, and $\varepsilon_{\mathcal{B}}\left(\mathbf{1}_{\mathcal{B}}\right)=1$;for all $x, y \in \mathcal{A}, \xi, \eta \in \mathcal{B}$, where $\mathbf{1}_{\mathcal{A}}, \mathbf{1}_{\mathcal{B}}$ are the identity elements of $\mathcal{A}$ and $\mathcal{B}$ respectively. Moreover a trivial braiding or twist map $\tau$ is defined in $\mathcal{A}$ as $\tau(x \otimes y)=y \otimes x$ while in $\mathcal{B}$ a braiding $\varphi$ ia defined as $\varphi(\xi \otimes \eta)=q \eta \otimes \xi$ where $q=e^{2 \pi i N}$. Random walks leading to diffusion equations have been carried out independently in $\mathcal{A}[4]$ and $\mathcal{B}$ [5, 6].

We shall now consider the case of performing a random walk on what we shall call a smash line which arises by first merging of the two algebras above. The merging of $\mathcal{A}$ and $\mathcal{B}$ as $\Omega=\mathcal{A} \otimes \mathcal{B}$ where $x$ is embeded as $x \otimes \mathbf{1}_{\mathcal{A}}$ and $\xi$ as $\mathbf{1}_{\mathcal{B}} \otimes \xi$, will eventually be formulated as an associative smash product algebra $\Omega$ which will also have a smash coproduct algebra structure. Explicitly the maps of product, coproduct, unit and counit in $\Omega$ are given by

$$
\mu_{\Omega} \equiv\left(\mu_{\mathcal{A}} \otimes \mu_{\mathcal{B}}\right) \circ\left(i d_{\mathcal{A}} \otimes \tau \otimes i d_{\mathcal{B}}\right), \Delta_{\Omega} \equiv\left(i d_{\mathcal{A}} \otimes \tau \otimes i d_{\mathcal{B}}\right) \circ\left(\Delta_{\mathcal{A}} \otimes \Delta_{\mathcal{B}}\right)
$$




$$
u_{\Omega}(1)=\mathbf{1}_{\Omega}, \varepsilon_{\Omega} \equiv \epsilon_{\mathcal{A}} \otimes \epsilon_{\mathcal{B}}, \text { with } \mathbf{1}_{\Omega}=\mathbf{1}_{\mathcal{A}} \otimes \mathbf{1}_{\mathcal{B}}
$$

We can now give a smash product and coproduct algebra structure [3, 8, 9] to $\Omega$ by means of the braiding map $\Psi: \Omega \otimes \Omega \rightarrow \Omega \otimes \Omega$ where

$$
\begin{gathered}
\Psi\left(x \otimes \mathbf{1}_{\mathcal{B}} \otimes \mathbf{1}_{\mathcal{A}} \otimes \xi\right)=Q\left(\mathbf{1}_{\mathcal{A}} \otimes \xi \otimes x \otimes \mathbf{1}_{\mathcal{B}}\right),(Q \in \mathbf{R}), \\
\Psi\left(\mathbf{1}_{\mathcal{A}} \otimes \xi \otimes \mathbf{1}_{\mathcal{A}} \otimes \eta\right)=q\left(\mathbf{1}_{\mathcal{A}} \otimes \eta \otimes \mathbf{1}_{\mathcal{A}} \otimes \xi\right), \\
\Psi\left(x \otimes \mathbf{1}_{\mathcal{B}} \otimes x^{\prime} \otimes \xi\right)=\left(x^{\prime} \otimes \mathbf{1}_{\mathcal{B}} \otimes x \otimes \mathbf{1}_{\mathcal{B}}\right),
\end{gathered}
$$

with product $\mu_{\Omega^{2}}=\left(\mu_{\Omega} \otimes \mu_{\Omega}\right) \circ\left(i d_{\Omega} \otimes \Psi \otimes i d_{\Omega}\right)$, coproduct $\Delta_{\Omega^{2}} \equiv\left(i d_{\Omega} \otimes \Psi \otimes i d_{\Omega}\right) \circ\left(\Delta_{\Omega} \otimes \Delta_{\Omega}\right)$, unit and counit as $u_{\Omega^{2}}(1)=\mathbf{1}_{\Omega^{2}}, \varepsilon_{\Omega^{2}} \equiv \epsilon_{\Omega} \otimes \epsilon_{\Omega}$. This product extended to $\Omega^{n}$ by the relation

$$
\mu_{\Omega^{n}}=\left(\mu_{\Omega^{n-1}} \otimes \mu_{\Omega}\right) \circ \prod_{k=1}^{2 n-3} \circ\left(i d_{\Omega}^{\otimes^{2 n-2-k}} \otimes \Psi \otimes i d_{\Omega}^{k}\right) .
$$

provides the algebra of increments of the random walk on the smash line i.e

$$
x_{i} x_{j}=x_{j} x_{i}, \forall i, j, \xi_{i} \xi_{j}=q \xi_{j} \xi_{i}, x_{i} \xi_{j}=Q \xi_{j} x_{i}, \text { fori }>j
$$

where the indices above indicate the position of the embeddings of $x$ and $\xi$ in the respective spaces (e.g. $\xi_{2} \xi_{1}=\left(\mathbf{1}_{\mathcal{A}} \otimes \mathbf{1}_{\mathcal{B}} \otimes \mathbf{1}_{\mathcal{A}} \otimes \xi\right)\left(\mathbf{1}_{\mathcal{A}} \otimes \xi \otimes \mathbf{1}_{\mathcal{A}} \otimes \mathbf{1}_{\mathcal{B}}\right)$

$\left.=q\left(\mathbf{1}_{\mathcal{A}} \otimes \xi \otimes \mathbf{1}_{\mathcal{A}} \otimes \mathbf{1}_{\mathcal{B}}\right)\left(\mathbf{1}_{\mathcal{A}} \otimes \mathbf{1}_{\mathcal{B}} \otimes \mathbf{1}_{\mathcal{A}} \otimes \xi\right)=q \xi_{1} \xi_{2}\right)$. In addition using eqs. (1) and (2)), the $n$-th fold coproduct on $x^{k} \otimes \xi^{l} \in \Omega$ is given by:

$$
\begin{aligned}
& \Delta_{\Omega}^{n-1}\left(x^{k} \otimes \xi^{l}\right)=\sum_{i_{1}+\cdots+i_{n}=k} \sum_{j_{1}+\cdots+j_{n}=k}\left(\begin{array}{ccc}
k & \\
i_{n} & \cdots & i_{n}
\end{array}\right)\left[\begin{array}{ccc}
l & \\
j_{n} & \cdots & j_{n}
\end{array}\right]_{q} \\
& \times x^{i_{1}} \otimes \xi^{j_{1}} \otimes \cdots \otimes x^{i_{n}} \otimes \xi^{j_{n}}
\end{aligned}
$$

where ingeneral $\Delta^{n}=\left(\Delta \otimes i d^{\otimes^{n-1}}\right) \circ \Delta^{n-1}=\left(i d^{\otimes^{n-1}} \otimes \Delta\right) \circ \Delta^{n-1}$, and the $q$-binomial coefficient is defined as $\left[\begin{array}{c}m \\ k l\end{array}\right]_{q}=\frac{[m]_{q} !}{[k]_{q} ![l]_{q} !}$.

Diffusion equation. Let $\phi$ be a linear functional from our algebra to $\mathbf{C}$, which corresponds to probability denisty element $\rho$ satisfing the following relations:

$$
\phi(f)=<f>_{\phi}=\int \rho f=<\phi, f>\in \mathbf{C} .
$$


It is assumed that $\phi$ lives in the dual space of $\Omega$ where the product is defined to be the usual convolution operation between probability density functions, by means of the following relations:

$$
\phi^{* n}(f)=<\phi^{\otimes n}, \Delta^{n-1}(f)>
$$

where $\Delta^{n} \equiv \Delta_{\Omega}^{n}$. If this is interpreted as the state of probability function of the random walk after $n$ steps, then the general state after an n-step walk evaluated on a general observable element

$$
f(x, \xi)=\sum_{k, l \in N} d_{k l} x^{k} \xi^{l}+\sum_{i \in Z_{+}} b_{i} x^{i}+\sum_{j \in Z_{+}} c_{j} \xi^{j}
$$

of $\Omega$ reads,

$$
\phi^{* n}(f(x, \xi))=(\phi * \phi * \ldots * \phi)(f(x, \xi))=\left(\phi^{\otimes^{n}}\right) \Delta^{n-1}(f(x, \xi)) .
$$

By virtue of eq.(3), (5) and making the choice $\rho(x, \xi)=\rho_{1}(x) \rho_{2}(\xi)$ where $\rho_{1}(x)=p_{1} \delta(x-$ $a)+\left(1-p_{1}\right) \delta(x+a), \rho_{2}(\xi)=p_{2} \delta(\xi-\theta)+\left(1-p_{2}\right) \delta(\xi+\theta)$ for the density functions of the random walk where as usual $p_{1}$ and $p_{2}$ are chosen probablilities, we conclude that with respect to the $x \xi$-monomials

$$
\begin{gathered}
<x^{k} \otimes \xi^{l}>_{\phi^{* n}}=\sum_{i_{1}+\cdots+i_{n}=k} \sum_{j_{1}+\cdots+j_{n}=k}\left(\begin{array}{ccc} 
& k \\
i_{n} & \cdots & i_{n}
\end{array}\right)\left[\begin{array}{ccc} 
& l \\
j_{n} & \cdots & j_{n}
\end{array}\right]_{q} \\
\times \prod_{l=1}^{n}<x^{i_{l}} \otimes \xi^{j_{l}}>_{\phi}
\end{gathered}
$$

where in general

$$
\begin{gathered}
<x^{i} \otimes \xi^{j}>_{\phi}= \\
{\left.\left[p_{1} e^{a D_{x}}+\left(1-p_{1}\right) e^{-a D_{x}}\right]\left[p_{2} e_{q}^{\theta D_{\xi}}+\left(1-p_{2}\right) e_{q}^{-\theta D_{\xi}}\right]\right|_{x, \xi=0}\left(x^{i} \xi^{j}\right)=\phi_{x}\left(x^{i}\right) \phi_{\xi}\left(\xi^{j}\right) .}
\end{gathered}
$$

Let us now compute the system after $n$ steps and its limit as $n \rightarrow \infty$. Using Taylors expansion, the form $\phi^{* n}$ for the case of monomials of $x^{m}, \xi^{t}$ and $x^{k} \xi^{l}$ reads as follows:

$$
\begin{aligned}
\phi^{* n}= & \phi_{x}^{* n} \phi_{\xi}^{* n}=\left[\varepsilon_{\Omega}+2 a\left(p_{1}-1 / 2\right) D_{x}+a^{2} / 2 ! D_{x}^{2}+\ldots\right]^{n} \\
& \times\left.\left[\varepsilon_{\Omega}+2 \theta\left(p_{2}-1 / 2\right) D_{\xi}+\theta^{2} /[2]_{q} ! D_{\xi}^{2}+\ldots\right]^{n}\right|_{x, \xi=0}
\end{aligned}
$$

and where $D_{x}=\partial / \partial_{x}, D_{\xi} f(\xi)=\frac{f(\xi)-f(\xi q)}{(1-q) \xi}$. Following [4], [5] we substitute $2 a\left(p_{1}-1 / 2\right)=$ $\frac{c_{1} t}{n}, 2 \theta\left(p_{2}-1 / 2\right)=\frac{c_{2} t}{n}, a^{2} / 2=\frac{\alpha_{1} t}{n}, \theta^{2} /[2]_{q}=\frac{\alpha_{2} t}{n}$ and then we take the limit $n \rightarrow \infty$ with $t$, $c_{1}, c_{2}, \alpha_{1}, \alpha_{2}$ fixed and $t=n \delta, \delta$ being the size of the step in time, to obtain the continue limit of random walk where the steps are viewed (44, [5]) as steps in time. This yields

$$
\phi^{\infty}(f)=\left.\left(e^{c_{1} t D_{x}+\alpha_{1} t D_{x}^{2}} f\right)\right|_{x=0}+\left.\left(e^{c_{2} t D_{\xi}+\alpha_{2} t D_{\xi}^{2}} f\right)\right|_{\xi=0}+\left.\left(e^{c_{1} t D_{x}+\alpha_{1} t D_{x}^{2}+c_{2} t D_{\xi}+\alpha_{2} t D_{\xi}^{2}} f\right)\right|_{x, \xi=0}
$$


where the limit $(1+z / n)^{n} \rightarrow e^{z}$ for $n \rightarrow \infty$ has been used. To obtain the diffusion equation we have, that for a general $f$ of the form (丰),

$$
\begin{aligned}
\int\left(\partial_{t} \rho^{\infty}\right) f & =\partial_{t} \phi^{\infty}(f)=\phi^{\infty}\left(\left(c_{1} D_{x}+\alpha_{1} D_{x}^{2}+c_{2} D_{\xi}+\alpha_{2} D_{\xi}^{2}\right) f\right) \\
& =\int\left(\left(-c_{1} D_{x}+\alpha_{1} D_{x}^{2}+c_{2} D_{\xi}^{*}+\alpha_{2} D_{\xi}^{* 2}\right) \rho^{\infty}\right) f
\end{aligned}
$$

which implies that

$$
\partial_{t} \rho^{\infty}=\left(-c_{1} D_{x}+\alpha_{1} D_{x}^{2}+c_{2} D_{\xi}^{*}+\alpha_{2} D_{\xi}^{* 2}\right) \rho^{\infty}
$$

with solution:

$$
\rho_{1}^{\infty}(a)=\left(4 \pi \alpha_{1} t\right)^{-1} e^{-\frac{\left(a-c_{1} t\right)^{2}}{4 \alpha_{1} t}}, \rho_{2}^{\infty}(\theta)=\sum_{k=0}^{N-1} \theta^{N-1-k} \sum_{l=0}^{l<k / 2} \frac{\left(c_{2} t\right)^{k-l}\left(\alpha_{2} / c_{2}\right)^{l}[k]_{q} !}{l !(k-2 l) !}
$$

Non Stationary Case. Consider the Hamiltonian evolution of a quantity F depending in general on the phase space variables $x, p, \xi, p_{\xi}$ where we have assumed that $\xi^{2}=0$ :

$$
\{F, H\}=\frac{\partial F}{\partial x} \frac{\partial H}{\partial p}-\frac{\partial F}{\partial p} \frac{\partial H}{\partial x}+(-1)^{\epsilon}\left(\frac{\partial F}{\partial \xi} \frac{\partial H}{\partial p_{\xi}}+\frac{\partial F}{\partial p_{\xi}} \frac{\partial H}{\partial \xi}\right)
$$

where $\epsilon=0,1$ is the degree of $F$. We can defined, using $t$ as a time parameter, the action of $e^{t V_{H}}$ on $x_{0}$ is given by $e^{t V_{H}} x_{0}=x_{t}$, and similarly $e^{t V_{H}} \xi_{0}=\xi_{t}$ where the action of $V_{H}$ isgenerally given by

$$
V_{H}(F)=\frac{\partial H}{\partial p} \frac{\partial F}{\partial x}-\frac{\partial H}{\partial x} \frac{\partial F}{\partial p}+(-1)^{\epsilon}\left(\frac{\partial H}{\partial p_{\xi}} \frac{\partial F}{\partial \xi}-\frac{\partial H}{\partial \xi} \frac{\partial F}{\partial p_{\xi}}\right)
$$

We can now evaluate $\phi\left(x_{t}^{k} \xi_{t}^{l}\right)$ using the choice of $\rho$ as mentioned underneath relation (5):

$$
\phi\left(x_{t}^{k} \xi_{t}^{l}\right)=\left[p_{1} e^{a_{t} D_{x}}+\left(1-p_{1}\right) e^{a_{t}^{\prime} D_{x}}\right]\left[p_{2} e^{\theta_{\tau} d_{\xi}}+\left(1-p_{2}\right) e^{\theta_{\tau}^{\prime} d_{\xi}}\right]_{\mid x, \xi=0} x_{0}^{k} \xi_{0}^{l}=\phi_{t}\left(x_{0}^{k} \xi_{0}^{l}\right)
$$

where we have set $a_{t}=a-t \lambda, a_{t}^{\prime}=-a-t \lambda, \theta_{t}=\theta-t \tilde{\lambda}$ and $\theta_{t}^{\prime}=-\theta-t \tilde{\lambda}, \lambda=\frac{\partial H}{\partial p}$, $\tilde{\lambda}=-\frac{\partial H}{\partial p_{\xi}}$. We can now expand the exponentials in the above relation and after some algebra which involves taking the limit $n \rightarrow \infty$ we find that

$$
\phi_{t}^{\infty}(f)=\left[e^{\left(c_{1}-\lambda d_{1}\right) t D_{x}+\alpha_{1} t D_{x}^{2}+\left(c_{2}-\tilde{\lambda} d_{2}\right) t D_{\xi}}\right](f)_{\mid x, \xi=0}=\left(e^{t K} f\right)_{\mid x, \xi=0}=\int \rho^{\infty} f=<\rho^{\infty}, f>
$$

where we have implemented the following substitutions $2 a\left(p_{1}-1 / 2\right)=\frac{c_{1} t}{n}, t \lambda=\lambda \frac{d_{1} t}{n}$, $\frac{a^{2}}{2}=\frac{\alpha_{1} t}{n}, 2 \theta\left(p_{2}-1 / 2\right)=\frac{c_{2} t}{n}, t \tilde{\lambda}=\tilde{\lambda} \frac{d_{2} t}{n},\left(d_{1}, d_{2}\right.$, real contsants $)$. 
Then the diffusion equation reads:

$$
\partial_{t} \rho^{\infty}=\left(-c_{1} D_{x}+\alpha_{1} D_{x}^{2}+c_{2} D_{\xi}^{*}+\lambda D_{x}-\tilde{\lambda} D_{\xi}^{*}\right) \rho^{\infty}
$$

where we have set $d_{1}=d_{2}=1$.

Matrix Realisation. We will now employ the $N$-dimentional matrix representations of $\xi$, as $(\xi)_{i, i+1}=1, D_{\xi}$ as $\left(D_{\xi}\right)_{i, i+1}=\{i\}$ and $D_{\xi}^{*}$ as $\left(D_{\xi}^{*}\right)_{i, i+1}=\{i\} e^{\omega^{-1}\{i\}}, i=1, \cdots, N-1$ and all other entries zero and where $\{x\}=\frac{1-\omega^{x}}{1-\omega}, \omega=e^{2 i \pi / N}$, that they have explicitely been constructed in [10, 7], to get a matrix realisaton for the stationary diffusion equation (10). In particular for the matrix representation of $D_{\xi}^{*}(t)$ one should take in to account [5] where the above has been obtained via the use of anyonic Leibnitz rule $D_{\xi}(f g)=$ $\left(D_{\xi} f\right) g+L_{q} f\left(D_{\xi} g\right)$ and the property that $D_{\xi}^{*}=-D_{\xi} L_{q^{-1}}$.Using this realization we can write $(10)$ as $\partial_{t} \rho^{\infty}=H \rho^{\infty}$, where

$$
H=\left(\begin{array}{lllll}
H_{x} & c_{2} \lambda_{1} & \alpha_{2} \lambda_{1} \lambda_{2} & 0 & 0 \\
0 & H_{x} & c_{2} \lambda_{2} & \alpha_{2} \lambda_{2} \lambda_{3} & 0 \\
\vdots & \vdots & \vdots & \vdots & \vdots \\
0 & 0 & 0 & H_{x} & c_{2} \lambda_{N-1} \\
0 & 0 & 0 & 0 & H_{x}
\end{array}\right)
$$

with $\lambda_{i}=\{i\} e^{\omega^{-1}\{i\}}, i=1, \cdots, N-1$, and $H_{x}=-c_{1} D_{x}+\alpha_{1} D_{x}^{2}$. Expanding a generic $\rho^{\infty}$ as:

$$
\rho^{\infty}(x, \xi, t)=\sum_{i=0}^{N-1} \sum_{j=0}^{\infty} \rho_{i j} x^{j} \xi^{i}=\sum_{i=0}^{N-1} \rho_{i}(x) \xi^{i}=\left(\begin{array}{llll}
\rho_{0}(x) & 0 & 0 & 0 \\
\rho_{1}(x) & \rho_{0}(x) & 0 & 0 \\
\vdots & \vdots & \vdots & \vdots \\
\rho_{N-1}(x) & \rho_{N-2}(x) & \ldots & \rho_{0}(x)
\end{array}\right)
$$

yields the following general system of differential equations to be solved:

$$
\frac{\partial \rho_{k}}{\partial t}=H_{x} \rho_{k}+c_{2} \lambda_{k+1} \rho_{k+1}+\alpha_{2} \lambda_{k+1} \lambda_{k+2} \rho_{k+2} \quad \text { for } \quad \mathrm{k}=0,1, \cdots, \mathrm{N}-1 .
$$

Conclusions. We have constructed an algebraic random walk and its associated limit governed by a diffusion equation on a space with real and paragrassmann components. The construction is based on the smashing of the algebra of functions of the underlined space. The algebraic approach is flexible and allows to determine statistical moments of the random walk and matrix realizations of its diffusion limit. Details of the smash line Brownian motion as well as extensions to random walks and diffusions on operator algebras of Quantum Mechanics can be found elsewere 11, 12] . 


\section{References}

[1] P. A. Meyer, Quantum Probability for Probabilists (Lect. Notes Math. 1538), (Springer, Berlin 1993).

[2] M. Schürmann, White Noise on Bialgebras (Lect. Notes Math. 1544), (Springer, Berlin 1993).

[3] S. Majid, Foundations of Quantum Groups Theory (Cambridge Univ. Press, 1955), ff. chapter 5 .

[4] S. Majid, Quantum random walks and time reversal, Int. J. Mod. Phys. 8 , 4521-4545 (1993).

[5] S. Majid, M. J. Rodriguez-Plaza, Random walk and the Heat Equation on Superspace and Anyspace, J. Math. Phys. 33, 3753-3760 (1994)

[6] U. Franz and R. Schott, Diffusion on Braided Spaces J. Math. Phys. 39, 2748 - 2762 (1998).

[7] A. P. Isaev, Paragrassmann Integral, Discrete Systems and Quantum Groups, $\mathrm{q}$ alg/9609030, 1996.

[8] S. Caenepeel, I. Bogdan, G. Militaru and S. Zhu, Factorization Structure of Algebras and Coalgebras, math.qa/9809063.

[9] Y. Bespalov and B. Drabant, Cross Product Bialgebras - Part I-II, math/9802028, math/9904142.

[10] M. Rausch de Traubenberg, Clifford Algebras of Polynomials, Generalized Grassmann Algebras and q-Deformed Heisenberg Algebras, Adv. Appl. Clifford Alg. 4, 131 (1994).

[11] D. Ellinas and I. Tsohantjis, Random Walks and Diffusions on a Smash Line, to appear.

[12] D. Ellinas, Quantum Diffusions and Appell Systems, TUC-preprint Nov. 1999. 